Article

\title{
Three New Integration Vectors and Fluorescent Proteins for Use in the Opportunistic Human Pathogen Streptococcus pneumoniae
}

\author{
Lance E. Keller ${ }^{\dagger}$, Anne-Stéphanie Rueff ${ }^{\dagger}$, Jun Kurushima ${ }^{\circledR}$ and Jan-Willem Veening $*$ \\ Department of Fundamental Microbiology, Faculty of Biology and Medicine, University of Lausanne, Biophore \\ Building, CH-1015 Lausanne, Switzerland; lanceedward.keller@unil.ch (L.E.K.); \\ anne-stephanie.rueff@unil.ch (A.-S.R.); jun.kurushima@unil.ch (J.K.) \\ * Correspondence: jan-willem.veening@unil.ch; Tel.: +41-(0)21-6925625 \\ † These authors contributed equally.
}

Received: 24 April 2019; Accepted: 20 May 2019; Published: 22 May 2019

check for updates

\begin{abstract}
Here, we describe the creation of three integration vectors, $\mathrm{PPEPX}, \mathrm{pPEPY}$ and $\mathrm{pPEPZ}$, for use with the opportunistic human pathogen Streptococcus pneumoniae. The constructed vectors, named PEP for Pneumococcal Engineering Platform (PEP), employ an IPTG-inducible promoter and BglBrick and BglFusion compatible multiple cloning sites allowing for fast and interchangeable cloning. PEP plasmids replicate in Escherichia coli and harbor integration sites that have homology in a large set of pneumococcal strains, including recent clinical isolates. In addition, several options of antibiotic resistance markers are available, even allowing for selection in multidrug resistant clinical isolates. The transformation efficiency of these PEP vectors as well as their ability to be expressed simultaneously was tested. Two of the three PEP vectors share homology of the integration regions with over half of the $S$. pneumoniae genomes examined. Transformation efficiency varied among PEP vectors based on the length of the homology regions, but all were highly transformable and can be integrated simultaneously in strain D39V. Vectors used for pneumococcal cloning are an important tool for researchers for a wide range of uses. The PEP vectors described are of particular use because they have been designed to allow for easy transfer of genes between vectors as well as integrating into transcriptionally silent areas of the chromosome. In addition, we demonstrate the successful production of several new spectrally distinct fluorescent proteins (mTurquoise2, mNeonGreen and mScarlet-I) from the PEP vectors. The PEP vectors and newly described fluorescent proteins will expand the genetic toolbox for pneumococcal researchers and aid future discoveries.
\end{abstract}

Keywords: Streptococcus pneumoniae; pneumococcal engineering platform; synthetic biology; expression vectors; mTurquoise2; mNeonGreen; mScarlet-I

\section{Introduction}

Infectious disease is one of the most common causes of death worldwide and is an object of intense scientific study [1]. A powerful tool into the research of human pathogens is the ability to manipulate the pathogen in a desired manner to observe the outcome. These manipulations can take many forms; gene deletions, genetic complementation, gene mutations, or controlled expression. To aid in alteration of a target genome, numerous methods have been developed over the years with varying success depending on the targeted pathogen [2]. Tool development in model organisms tend to be over represented compared to other species, with bacterial human pathogens having fewer options. While the study of model organisms has led to great advances in understanding the nature of diverse bacterial functions, they are not useful for pathogen specific processes. In an attempt to ameliorate this 
deficit, we set out to develop several cloning vectors useful for genetic manipulation in Streptococcus pneumoniae, even in recent clinically relevant isolates.

The bacterium S. pneumoniae is an important opportunistic human pathogen that is responsible for over a million deaths per annum [3]. S. pneumoniae has been extensively studied in the past decade and several useful tools for genetic manipulation have been created that allow for stable single copy integration at the genome. However, there is still a lack of robust suicide vectors that do not disrupt native expression of important genes that can be combined to insert several genes in the same strain or can be applied to multi-drug resistant clinical isolates. One of the most commonly used ectopic integration site in S. pneumoniae is the bgaA locus [4-7], which disrupts this $\beta$-galactosidase, which might have an important function during colonization of human epithelial cells [8-10]. The only neutral integration site for which integration plasmids have been developed is the CEP locus (chromosomal expression platform), which is a transcriptionally silent site within a truncated IS 1167 element downstream of the well-studied ami operon [11,12]. However, this loci is not very well conserved among pneumococcal strains (see Results below).

Utilizing extensive RNA-seq data for S. pneumoniae in multiple conditions [13], transcriptionally silent regions were identified for potential chromosomal locations to use as recombination sites. Using the pneumococcal engineering platform (PEP) vector as a guide [12], novel integration vectors were developed for use at these regions. In this study, we have developed and tested three new PEP vectors, pPEPX, pPEPY, and PPEPZ, for their ability to be transformed into S. pneumoniae, compatibility for co-expression, and frequency of occurrence in pneumococcal isolates. In addition, three spectrally distinct and intrinsically bright fluorescent proteins, mTurquoise2, mNeonGreen, and mScarlet-I, were added to the fluorescence protein toolbox now available for S. pneumoniae.

\section{Materials and Methods}

\subsection{Growth Conditions}

All pneumococcal strains are derived from the parent strain D39V [14] and were grown in the semi-synthetic media C + Y formulation 2018 [15] under selection conditions where applicable, see Table 1. Standard pneumococcal transformation [16] protocols using synthetic CSP were used for strain construction with selection on Columbia agar supplemented with $2 \%$ sheep blood and appropriate antibiotics. Plasmids were cloned and maintained in Escherichia coli strains XL1-Blue or Stbl3 with LB media and selected on LB agar plates with appropriate antibiotics (Table 1). Transformation into E. coli was done using rubidium chloride chemically competent cells with standard procedures. Induction of all strains containing an isopropyl $\beta$-D-1-thiogalactopyranoside (IPTG)-inducible promoter were activated with $1 \mathrm{mM}$ of IPTG for $1 \mathrm{~h}$ before visualization by microscopy.

Table 1. List of constructed plasmids. Spec-spectinomycin, Kan-Kanamycin, Gent-Gentamycin, Erm-Erythromycin, Amp-Ampicillin. Tmp-Trimethoprim.

\begin{tabular}{|c|c|c|c|}
\hline Plasmid Name & Features & Selection-E. coli & Selection-S. pneumoniae \\
\hline pPEPX & $\begin{array}{l}\text { Promoterless integrative plasmid with } \\
\text { terminator }\end{array}$ & Spec $50 \mu \mathrm{g} / \mathrm{mL}$ & Spec $200 \mu \mathrm{g} / \mathrm{mL}$ \\
\hline pPEPX-P 1 lac & $\begin{array}{l}\text { Integrative plasmid with IPTG inducible } \\
\text { promoter }\end{array}$ & Spec $50 \mu \mathrm{g} / \mathrm{mL}$ & Spec $200 \mu \mathrm{g} / \mathrm{mL}$ \\
\hline pPEPY & $\begin{array}{c}\text { Promoterless integrative plasmid with } \\
\text { terminator }\end{array}$ & Kan $50 \mu \mathrm{g} / \mathrm{mL}$ & Kan $250 \mu \mathrm{g} / \mathrm{mL}$ \\
\hline pPEPY-P lac & $\begin{array}{l}\text { Integrative plasmid with IPTG inducible } \\
\text { promoter }\end{array}$ & Kan $50 \mu \mathrm{g} / \mathrm{mL}$ & Kan $250 \mu \mathrm{g} / \mathrm{mL}$ \\
\hline pASR100 (pPEPZ-P lac $)$ & $\begin{array}{l}\text { Integrative plasmid with IPTG inducible } \\
\text { promoter }\end{array}$ & Spec $50 \mu \mathrm{g} / \mathrm{mL}$ & Spec $200 \mu \mathrm{g} / \mathrm{mL}$ \\
\hline
\end{tabular}


Table 1. Cont.

\begin{tabular}{|c|c|c|c|}
\hline Plasmid Name & Features & Selection-E. coli & Selection-S. pneumoniae \\
\hline pASR102 (pPEPZ-P lac $)$ & $\begin{array}{l}\text { Integrative plasmid with IPTG inducible } \\
\text { promoter }\end{array}$ & Gent $40 \mu \mathrm{g} / \mathrm{mL}$ & Gent $50 \mu \mathrm{g} / \mathrm{mL}$ \\
\hline pASR103 (pPEPZ-P lac $)$ & $\begin{array}{l}\text { Integrative plasmid with IPTG inducible } \\
\text { promoter (Amp selection for E. coli) }\end{array}$ & Amp 100 нg/mL & Erm $0.5 \mu \mathrm{g} / \mathrm{mL}$ \\
\hline pASR130 (pPEPZ-P lac $)$ & $\begin{array}{l}\text { Integrative plasmid with IPTG inducible } \\
\text { promoter (Amp selection for E. coli) }\end{array}$ & Amp $100 \mu \mathrm{g} / \mathrm{mL}$ & Tmp $10 \mu \mathrm{g} / \mathrm{mL}$ \\
\hline pPEPY-P ${ }_{\text {lac }}$-Link-mTurquoise2 & $\begin{array}{l}\text { Integrative plasmid with IPTG inducible } \\
\text { promoter and linker for C-terminal fusion } \\
\text { of mTurquoise } 2\end{array}$ & Kan $50 \mu \mathrm{g} / \mathrm{mL}$ & Kan $250 \mu \mathrm{g} / \mathrm{mL}$ \\
\hline pPEPY-P lac-Link-mNeonGreen & $\begin{array}{l}\text { Integrative plasmid with IPTG inducible } \\
\text { promoter and linker for C-terminal fusion } \\
\text { of mNeonGreen }\end{array}$ & Kan $50 \mu \mathrm{g} / \mathrm{mL}$ & Kan $250 \mu \mathrm{g} / \mathrm{mL}$ \\
\hline pPEPY-P lac - Link-mScarlet-I & $\begin{array}{l}\text { Integrative plasmid with IPTG inducible } \\
\text { promoter and linker for C-terminal fusion } \\
\text { of mScarlet-I }\end{array}$ & Kan $50 \mu \mathrm{g} / \mathrm{mL}$ & Kan $250 \mu \mathrm{g} / \mathrm{mL}$ \\
\hline
\end{tabular}

\subsection{Plasmid Construction}

RNA-seq data available at https://veeninglab.com/pneumoexpress [13] and gene annotation data at https://veeninglab.com/pneumobrowse [14] was used for selection of transcriptionally silent regions.

pPEPX. This vector is a modified smaller version of pPEP1 [12] lacking the chloramphenicol resistance marker and containing the high copy number origin of replication from pUC18, but still integrates at the neutral CEP locus near amiF [11]. The pUC18 origin of replication was amplified from pLA01 [17] using primers pUC18-F-KpnI and pUC18-R-NotI. The amplified fragment containing pUC18 origin and pPEP1 were digested with KpnI and NotI (NEB, Ipswich, MA, USA) for $1 \mathrm{~h}$ at $37^{\circ} \mathrm{C}$ followed by overnight RT ligation with T4 DNA ligase (ThermoScientific, Waltham, MA, USA). The resulting plasmid was then digested with KpnI and NcoI (NEB, Ipswich, MA, USA) for $1 \mathrm{~h}$ at $37^{\circ} \mathrm{C}$ and ligated with hybridized primers NcoI-KpnI-link-F and NcoI-KpnI-link-R that were similarly digested. The IPTG inducible promoter was added to the promoterless pPEPX plasmid by digestion of pPEP8PL8-2 [18] with EcoRI and BglII and ligating appropriate fragment to complementary digested $\mathrm{PPEPX}$.

pPEPY-Creation of plasmid was completed through Gibson Assembly (NEB, Ipswich, MA, USA) using a minimum of $20 \mathrm{bp}$ overlapping regions of five DNA fragments following manufacturers protocol. Integration regions were amplified from D39V genomic DNA using primer pairs LK042/LK043 and LK046/LK047. Origin of replication and multiple cloning site, which contains transcriptional terminator within the site, was amplified from pPEPX vector using primer pairs LK044/LK045 and LK040/LK041 respectively. Kanamycin resistance cassette was amplified from plasmid pDG783 [19] with primer pair LK033/LK034 that included addition of the $\mathrm{P}_{\mathrm{F} 6}$ promoter [12] for kanamycin expression. Three terminators were added after the multiple cloning site to this vector through Gibson assembly of terminators from plasmid pJWV100 [7] (OVL68/69) and the pre-pPEPY plasmid (OVL66/67), creating pPEPY. The IPTG inducible promoter $\left(\mathrm{P}_{\text {lac }}\right)$ [20] was added through restriction digestion of $\mathrm{P}_{\text {lac }}$ amplified from FtsK-mNeonGreen (van Raaphorst and Veening, unpublished) with primer pair

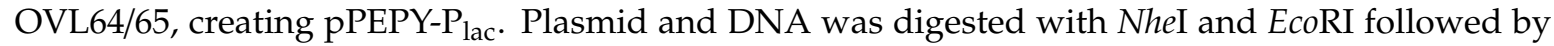
ligation and transformation into E. coli strain XL1-Blue. The PPEPY plasmid integrates at chromosomal integration locus (CIL) thereby interrupting the non-essential pseudogene spv_2165.

pPEPZ-Creation of an integration vector of S. pneumoniae at the locus spv_2417 (aka spd_1735) was completed through Golden Gate cloning. Primers were designed with Benchling to amplify four different DNA fragments. Primer pairs OVL681/682 and OVL683/684 were used to amplify the downstream and upstream homologous region, respectively, for integration into S. pneumoniae by using chromosomal DNA of D39V as template. Primers OVL685/686 were used to amplify the pUC18 origin of replication of from pPEPX vector and primers OVL687/688 were used to amplify a fragment containing the promoter $\mathrm{P}_{\text {lac }}$, the multiple cloning site, and the spectinomycin marker from 
the pPEP1-P lac vector [12]. DpnI treatment was performed for $15 \mathrm{~min}$ at $37{ }^{\circ} \mathrm{C}$ and the four different amplified parts were purified and then digested with BsmBI (NEB, Ipswich, MA, USA) enzyme at $55^{\circ} \mathrm{C}$ for $1.5 \mathrm{~h}$. This reaction was purified and ligated at RT for $1.5 \mathrm{~h}$ then transformed into E. coli XL1-Blue. Digestion control followed by sequencing was performed to confirm the construct. The pPEPZ vector integrates at the PEPZ Integration Position (ZIP) locus upstream of spv_1736 thereby interrupting the non-essential pseudogene spv_2417.

To generate the pPEPZ variants with gentamycin (gent) resistance, a Golden Gate strategy was employed. Primer pair OVL1066/1067 was used to amplify pPEPZ-P lac $_{\text {an }}$ and primers pair OVL1068/1069 was used to amplify the gent gene from D39V containing the lacI gene at the prs1 locus as template [20]. The two PCR products were purified, digested with BbsI-HF (NEB, Ipswich, MA, USA) at $37{ }^{\circ} \mathrm{C}$ for 1 $\mathrm{hr}$, and ligated at RT for $1 \mathrm{~h}$. Ligation mixture was used to transform E. coli strain Stbl3, sequencing was performed to confirm the construct called pASR102.

pPEPZ containing bla-First, $\beta$-lactamase gene (bla) was cloned into the pPEPZ-Plac (spec) vector in addition to the spectinomycin (spec) marker. Primer pairs OVL1190/1191 was used to amplified the bla gene from pASR113 as template (a pLA18 derivative, Rueff and Veening unpublished, [17]). The purified PCR fragment and pPEPZ-P lac vector were digested for $1.5 \mathrm{~h}$ at $37^{\circ} \mathrm{C}$ with NotI (NEB, Ipswich, MA, USA) to introduce the bla gene at the unique NotI site of pPEPZ-P $\mathrm{P}_{\text {lac }}$ (spec), between the origin of replication of pUC18 and the upstream homologous region. Fragments were ligated at RT for $2 \mathrm{~h}$ and transformed into E. coli strain Stbl3 to generate intermediate plasmid pASR101. Second, this plasmid was used for resistance marker exchange of the spectinomycin marker with an erythromycin (erm) marker by Golden Gate cloning. For that, pASR101 was amplified by PCR with primers OVL1066 and OVL1067, and the erm gene was amplified with primers OVL1070 and OVL1071 using strain XL39 (Liu and Veening unpublished) as template. The two PCR products were treated with DpnI (NEB, Ipswich, MA, USA) for $30 \mathrm{~min}$ at $37^{\circ} \mathrm{C}$, followed by purification and then digested with BbsI-HF (NEB, Ipswich, MA, USA) enzyme for $1 \mathrm{~h}$ at $37^{\circ} \mathrm{C}$. Reaction was purified and ligated for $30 \mathrm{~min}$ at room temperature and used to transform E. coli strain Stbl3. Sequencing was performed to confirm the construct called pASR103. Sequencing revealed an insertion between the erm marker and the $P_{\text {lac }}$ region. This insertion of $777 \mathrm{bp}$ is from E. coli chromosomal DNA and does not affect the functioning of the plasmid, as the $P_{\text {lac }}$ promoter is still well insulated by the transcriptional terminators, but does increase plasmid size.

Another version of the pPEPZ plasmid has been generated by replacing the erm marker with a trimethoprim (tmp) antibiotic marker. The plasmid was obtained by Golden Gate cloning as follows: primers OVL1696/1697 were used to amplify pASR103 and then DpnI (NEB, Ipswich, MA, USA) treated for $1 \mathrm{~h}$ at $37^{\circ} \mathrm{C}$. Primers OVL1698/1699 were used to amplify the tmp gene from pneumococcal strain AM39 [21] as template. Thereafter, the two PCR products were purified and then digested with AarI (NEB, Ipswich, MA, USA) enzyme at $37^{\circ} \mathrm{C}$ for $5 \mathrm{~h}$. After purification, the two parts were ligated at room temperature (RT) for $2 \mathrm{~h}$. The ligation mixture was used to transform E. coli strain Stbl3, and subsequent sequencing was performed to confirm the construct called pASR130. This plasmid also contains the E. coli chromosomal DNA insertion of $777 \mathrm{bp}$ in between tmp and the $\mathrm{P}_{\text {lac }}$ promoter region, since it is based on the pASR103 plasmid.

All plasmids were verified by sequencing and deposited at Addgene (plasmid\# 122631-122641).

Three additional pPEPY-P $\mathrm{P}_{\text {lac }}$ plasmids were created for carboxy(C)-terminal fusions of three spectrally distinct fluorescent proteins, mTurquoise2 [22], mNeonGreen [23], and mScarlet-I [24].

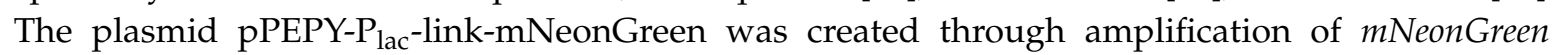
containing an 11 amino acid C-terminal linker with primers link-mNG-F and link-mNG-R from plasmid pRRneon, which contains a S. pneumoniae codon optimized version of $m$ NeonGreen (Raaphorst and Veening, unpublished). The resulting fragment was digested with BamHI and XhoI (NEB, Ipswich, MA, USA) for $1 \mathrm{~h}$ at $37^{\circ} \mathrm{C}$ followed by overnight RT ligation with similarly digested pPEPY-Plac plasmid and T4 DNA ligase (ThermoScientific, Waltham, MA, USA). The resulting plasmid was used for the template for pPEPY-P lac-link-mTurquoise2 using primers OVL914/915. mTurquoise2 was amplified 
with OVL916/917 using Addgene plasmid \#54844 (mTurquoise2-pBAD) as template. Subsequent DNA fragments were digested in a single reaction with BsmBI (NEB, Ipswich, MA, USA) for $1 \mathrm{~h}$ at $55{ }^{\circ} \mathrm{C}$ followed by overnight ligation at RT with T4 DNA ligase (ThermoScientific, Waltham, MA, USA). The mScarlet gene was obtained through S. pneumoniae codon optimized gene synthesis by IDT (Integrated DNA Technologies) followed by cloning into pPEPY-P lac. The $m S$ carlet-I variant

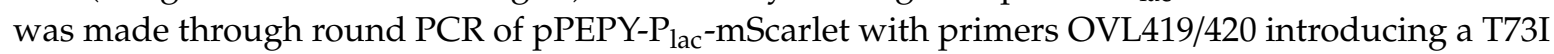
point mutation to the protein. Subsequent amplification was digested with $D p n \mathrm{I}$ (NEB, Ipswich, MA, USA) for $1 \mathrm{~h}$ at $37^{\circ} \mathrm{C}$ followed by treatment with ClonExpress (Vazyme, Nanjing, China) following manufacturers protocol. The $m S$ carlet-I variant was amplified from the resulting plasmid with OVL908/909 and combined with linearized pPEPY-P lac-Link (OVL906/907) and treated as for pPEPY-P lac-link-mTurquoise 2 creation. All ligation products were subsequently cloned into XL1-Blue and verified by sequencing. Addgene is pending accession number.

Gene fusions for ectopic integration at various PEP loci were created as follows. The fusion containing ComEA and mTurquoise2 was created through comEA amplification from D39V gDNA with primers OVL576/615. Amplification was digested with EcoRI and BamHI (NEB, Ipswich, MA, USA) for $1 \mathrm{~h}$ at $37^{\circ} \mathrm{C}$ and ligated with similarly digested pPEPY-P lac-Link-mTurquoise2 followed by overnight ligation at RT with T4 DNA ligase (ThermoScientific, Waltham, MA, USA). The HlpA and mNeonGreen fusion in pASR103, pPEPZ plasmid with erythromycin selection in S. pneumoniae, was created by hlpA-mNeonGreen amplification from strain VL1978 (Veening, unpublished) with primers OVL1569/1570. The resulting fragment and pASR103 were digested with BglII and XhoI (NEB, Ipswich, MA, USA) for $1 \mathrm{~h}$ at $37^{\circ} \mathrm{C}$ followed by overnight ligation at RT with T4 DNA ligase. CEP locus integration of spv_1159-mScarlet-I was created through amplification of VL1785 gDNA, which contains CEP integration with a spv_1159-sfGFP fusion, (Kurushima and Veening unpublished) with primer pairs OVL037/869 and OVL040/870. The fragment containing $m$ Scarlet-I was amplified from plasmid pPEPY-P lac-Link-mScarlet-I with primer pair OVL871/872. The resulting three fragments were digested together with BsmBI (NEB, Ipswich, MA, USA) for $1 \mathrm{~h}$ at $55^{\circ} \mathrm{C}$ followed by overnight ligation at RT with T4 DNA ligase. All oligos are listed in Supplementary Table S1.

\subsection{Strain Construction}

Parental strain D39V was transformed with a constitutively expressed LacI under gentamycin selection, downstream of the prs1 locus [20], leading to strain VL236. This strain was used for all future transformations, with the exception of plasmid pASR102, which was transformed into D39V, due to gentamycin selection of the plasmid. Standard pneumococcal transformation procedure was used to integrate all plasmids independently with appropriate selection [16]. For the strain expressing all plasmids (strain VL2706), each ligation product was sequentially cloned with verification of appropriate integration between each transformation. Double crossover by homologous recombination was tested after transformation of plasmids into D39V chromosome using primer pairs located outside the integration regions. For integration of PPEPX primer pair OVL873/OVL874 was used, for pPEPX integration primer pair LK352/LK353 was used and for PPEPZ integration primer pair OVL1004/OVL1005 was used.

\subsection{Database BLAST}

Basic Local Alignment Search Tool (BLAST) was done using NCBI blast-2.7.1+ against whole genomes with a minimum assembly at the contig level. All available S. pneumoniae sequences were downloaded from the NCBI database and combined with the Maela database (5029 strains). Genomes of closely related species S. pseudopneumoniae (43 strains) and S. tigurinus (19 strains) as well as other streptococcal species S. mutans (190 strains), S. equi (259 strains), S. pyogenes (449 strains), and S. suis (1261 strains) were downloaded from the NCBI database. Integration regions were used as the query sequences against BLAST databases. Results were filtered to include matches with over $95 \%$ sequence homology as well as containing at least $90 \%$ of the queried DNA. Duplicate matches were removed and 
results of both integration regions per plasmid were combined. A positive match was only identified if the combined filtered results included two hits per strain, one for upstream integration region and one for downstream integration region. Genetic circular map and comparative alignments were generated using GenomeMatcher and arcWithColor software with modification in graphic representation [25].

\subsection{Transformation Assay}

Wild type S. pneumoniae strain $\mathrm{D} 39 \mathrm{~V}$ was grown to $\mathrm{OD}_{600} 0.1$, washed and suspended in pre-warmed non-acidic $\mathrm{C}+\mathrm{Y}$ media. Competence was activated with $50 \mathrm{ng} / \mathrm{mL}$ of CSP- 1 at $37^{\circ} \mathrm{C}$ for $10 \mathrm{~min}$ and $45 \mu \mathrm{L}$ was added to a 96 well plate. Five microliters of DNA was added at various concentrations as indicated and incubated for $120 \mathrm{~min}$ at $37^{\circ} \mathrm{C}$. Samples were serially diluted and plated on non-selective blood agar (BA) and BA with the appropriate antibiotics. Transformation efficiency was calculated by taking the difference between the CFU of the non-selective and the selective BA. For transformation of strains other than D39V, the above protocol was followed with the addition of $50 \mathrm{ng} / \mathrm{mL}$ of CSP-2 for initial competence activation due to variations in ComD specificity.

\subsection{Microscopy}

Fluorescence microscopy was performed on a Leica DMi8 through a 100x phase contrast objective (NA 1.40) with a SOLA Light Engine (lumencor) light source. Light was filtered through external excitation filters 430/24 nm (Chroma ET430/24x), 470/40 nm (Chroma ET470/40x), and 545/25 nm (Chroma ET545/25x) for visualization of mTurquoise2, mNeonGreen, or mScarlet-I, respectively. For mTurquoise2, light passed through a cube (Leica 11536022) containing a polychroic mirror 455/520/595 nm while mNeonGreen and mScarlet-I used a cube (Leica 11536022) with a GFP/RFP polychroic mirror $(498 / 564 \mathrm{~nm})$. External emission filters used were from Chroma and ET470/24m, ET520/40m and ET605/70m were used for mTurquoise2, mNeonGreen and mScarlet-I, respectively. Exposure times of $700 \mathrm{~ms}, 200 \mathrm{~ms}$, and $500 \mathrm{~ms}$ with 100\% of light from SOLA Light Engine were performed for mTurquoise2, mNeonGreen, and mScarlet-I fusions respectively. Note that we also routinely image mNeonGreen through YFP filters (Chroma ET500/20x for excitation, Chroma ET535/30m for emission with a CFP/YFP/mCherry (455/520/595) polychroic mirror) in case there is signal bleed-through from the mTurquoise2 label in the GFP channel. Images were captured using LasX software (Leica) and exported to ImageJ [26] for final preparation.

\section{Results}

\subsection{Design and Construction of $p P E P X, p P E P Y$ and $p P E P Z$}

As seen in Figure 1, the three pneumococcal engineering platform plasmids created have compatible cloning sites following the BglBrick and BglFusion principle [12,27], to facilitate easy exchange between the plasmids. The exception is in the pPEPY vector with the EcoRI site located downstream of the $\mathrm{P}_{\text {lac }}$ promoter as opposed to upstream as in the other two plasmids. The integration regions vary for each vector, but are in transcriptionally silent areas of the genome as determined by RNA-seq data [13]. The pPEPX vector integrates about $295^{\circ}$ around the chromosome between the treR (spv_1665) and amiF (spv_1666) genes (Figure 2A,B). The pPEPY vector integrates at $75^{\circ}$ around the chromosome between $s p v \_2165$ and spv_2166, both encoding hypothetical proteins, (Figure 2A,B). The last constructed vector, $\mathrm{pPEPZ}$, integrates at $304^{\circ}$ around the chromosome, upstream of $s p v \_1736$, which encodes a degenerative hypothetical protein (Figure 2A,B). Transcriptional terminators flank the region containing the $P_{\text {lac }}$ promoter and multiple cloning site (MCS) in all vectors to ensure that the inserted DNA is not influenced by transcriptional events happening elsewhere on the chromosome. The origin of replication used for all plasmids is a high copy number E. coli origin derived from pUC18 that is not recognized by S. pneumoniae. All selective markers are expressed in both E. coli and S. pneumoniae. 


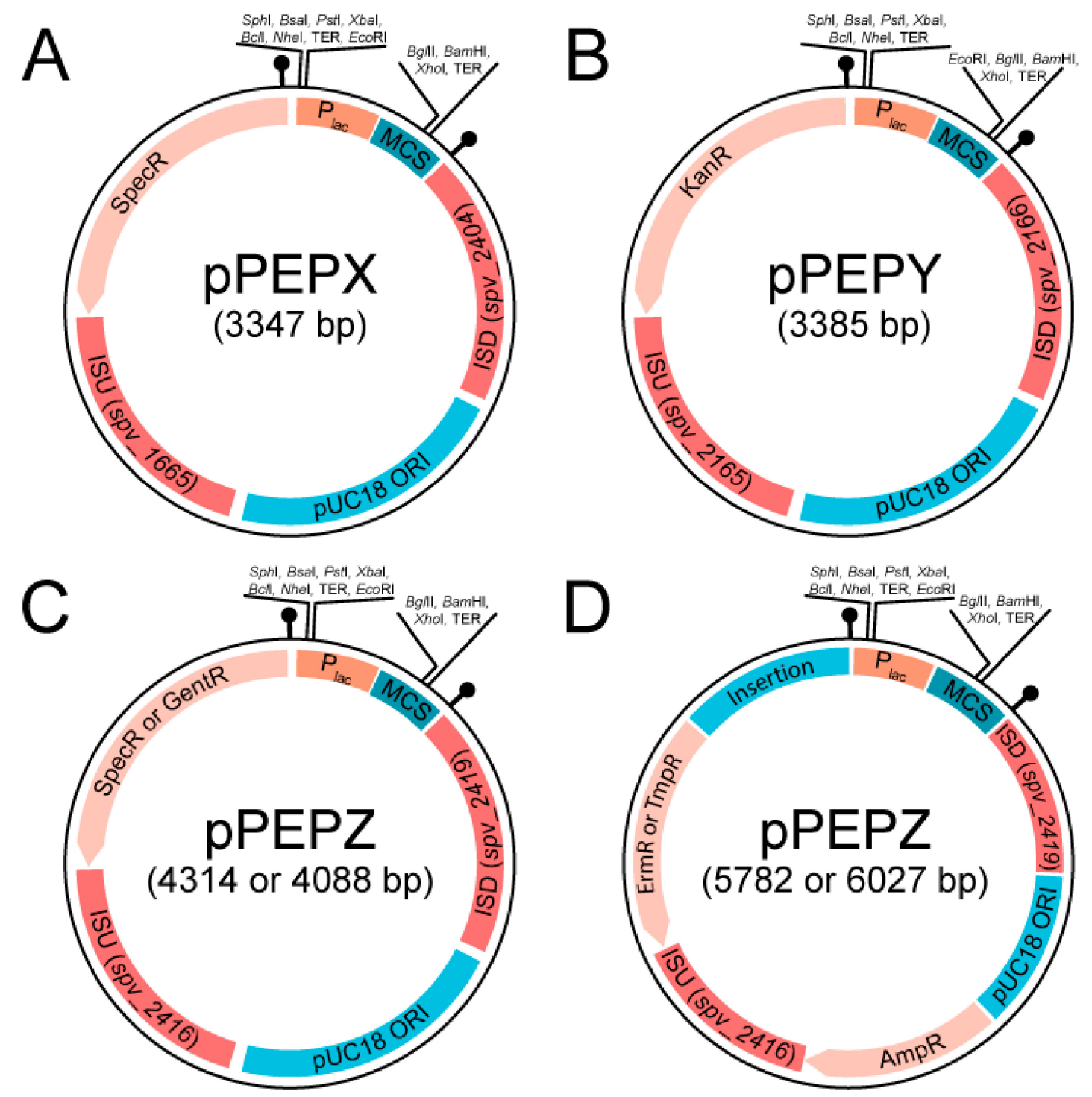

Figure 1. Diagram of general layout for various constructed PEP vectors. All PEP vectors have the same IPTG inducible promoter as well as the same pUC18 origin of replication which is permissive in E. coli, but not in S. pneumoniae. All vectors have a multiple cloning site featuring the BglBrick cloning method and integration regions that are transcriptionally silent based on RNA-seq data. Each promoter and MCS is flanked by strong terminators. (A) The pPEPX vector has a spectinomycin resistance marker and integration regions of approximately $500 \mathrm{bp}$ in length and integrates at $295.4^{\circ}$ around the D39V chromosome near amiF (spv_1667). (B) The pPEPY vector has a kanamycin resistance marker and integration regions of approximately $500 \mathrm{bp}$ in length and integrates at $74.6^{\circ}$ around the D39V chromosome near spv_0422. (C) Two PPEPZ vectors have either a spectinomycin or gentamycin resistance marker and integration regions of approximately $1000 \mathrm{bp}$ in length and integrates at $303.9^{\circ}$ around the D39V chromosome near spv_1736. (D) Two pPEPZ vectors have either an erythromycin or trimethoprim resistance marker along with an ampicillin marker for selection in E. coli. A nonfunctional E. coli chromosomal sequence insertion between the S. pneumoniae selection marker and the promoter is indicated. 


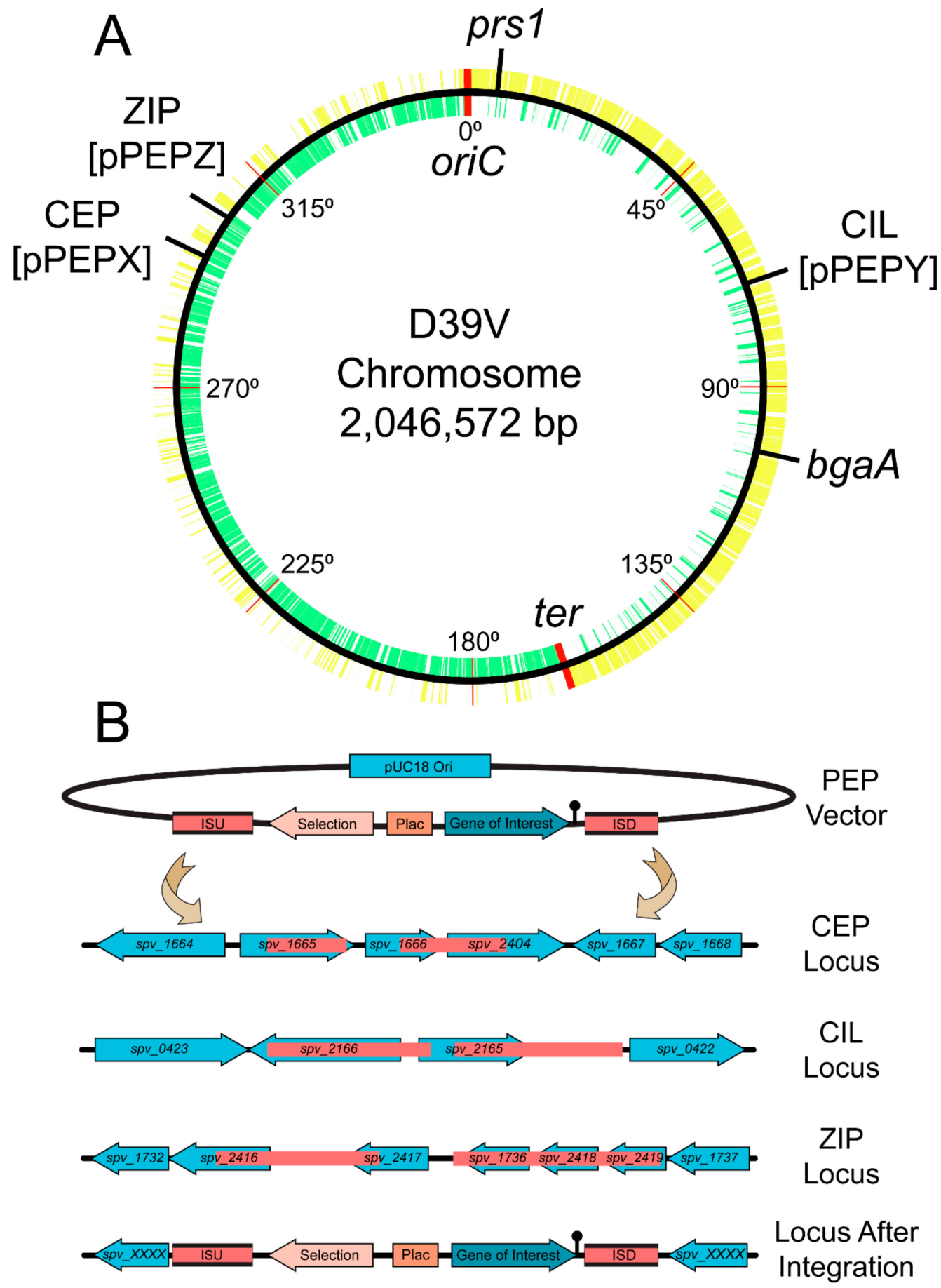

Figure 2. Chromosomal location of integration loci and integration schematic. (A) D39V chromosome with all positive strand genes (yellow), negative strand genes (green), and labeled integration loci. Position around the chromosome is demarcated every 45 degrees and origin of replication and terminus are indicated in red. Note that strain D39V has a large inversion around the ter region compared to laboratory strain R6 [14]. (B) Representation of insertion sequence upstream (ISU) and insertion sequence downstream (ISD) in pink for available PEP vectors. Double crossover event leads to stable integration in CEP (chromosomal expression platform, pPEPX), CIL (chromosomal integration locus, pPEPY), or ZIP (PEPZ Integration Position, pPEPZ) locus at indicated chromosomal locations based on most recent $\mathrm{D} 39 \mathrm{~V}$ genome annotation. 
Various antibiotic markers were created for pPEPZ to facilitate integration of multiple PEP vectors simultaneously, as well as providing multiple options depending on the need of the researcher. As pPEPZ has a large pneumococcal host range (see below), we also constructed pPEPZ variants with antibiotic resistance alleles less commonly found in clinical isolates such as trimethoprim and gentamycin (Figure 2). No PEP vectors contain the tetracycline resistance marker for selection, allowing strains to utilize PEP vectors in combination with the popular pPP1/pJWV/pKB bgaA-based integration vectors [5-7]. Table 1 summarizes vectors and available resistance markers.

Vector construction was done through various cloning techniques, but common motifs were kept for all PEP vectors. These include the same origin of replication for a high copy number in $E$. coli, similar multiple cloning sites to facilitate rapid exchange between plasmids, an IPTG inducible promoter with tight expression control, and multiple antibiotic selection markers for use in almost any background. Expression without an inducible promoter can be done using PPEPY or standard cloning can be used to exchange the promoter $\mathrm{P}_{\text {lac }}$ with any desired promoter. A list of available vectors and the selectable markers can be found in Table 1. Note that to make use of the $P_{\text {lac }}$ IPTG inducible promoter (Sorg et al. in preparation), the pneumococcal strain being transformed also needs to encode the LacI repressor from E. coli otherwise the promoter will be constitutively expressed. We noticed significant vector stability when leaving out the gene encoding the lacI repressor from the plasmid. Therefore, we used a separate, ectopic integration of lacI [20]. This lacI construct can be obtained via Addgene (catalog \#85589) and is integrated at the prs1 locus by selecting for gentamycin [20].

\subsection{High Transformation Efficiency of $p P E P X, p P E P Y$ and $p P E P Z$}

Transformation efficiency of these three vectors was determined in S. pneumoniae strain D39V (Figure 3). Multiple concentrations of DNA were tested and the amounts used were normalized to molar concentrations to remove concentration effects due to size differences in the plasmids. It was found that PPEPZ had higher transformation efficiencies than either PPEPX or PPEPY at all DNA concentrations tested $(0.064,0.32,1.6,8.0,40.0,200.0,1000.0 \mathrm{nM})$, with a maximum transformation efficiency of approximately $10^{-3}$ compared to $10^{-4}$. This is most likely due to the longer regions of homology present on PPEPZ (1 kb) compared to the homologous integration regions present on pPEPX (500 bps) and pPEPY (500 bps). For all vectors tested there was a two-log increase in transformation efficiency from the lowest concentration $(0.064 \mathrm{nM})$ of DNA added to the highest concentration (1000 nM). A rapid increase in transformation efficiency was observed upon increasing DNA concentrations, but gradually leveled off at a concentration of $40 \mathrm{nM}$ of DNA for all PEP vectors. These data are completely in line with previously described transformation efficiencies in S. pneumoniae that demonstrated a similar relation between length of the homology regions and DNA concentration [28,29]. Frequency of double crossover recombination was also determined for each vector by amplification of integration regions with oligos located outside of the integration regions included in the PEP vectors. This is an important consideration to verify correct integration and ensure removal or the vectors necessary for maintenance in E. coli, i.e., origin of replication and ampicillin resistance marker. Out of 10 colonies screened for double crossover recombination of each vector in D39V we found a $100 \%$ efficiency at integration at the correct locus by double crossover, even without linearizing the vector prior to transformation (Supplementary Figure S1). In summary, all described vectors can be efficiently transformed through double crossover events into strain D39V and acquisition of one vector does not exclude integration of other PEP vectors at available integration loci. 


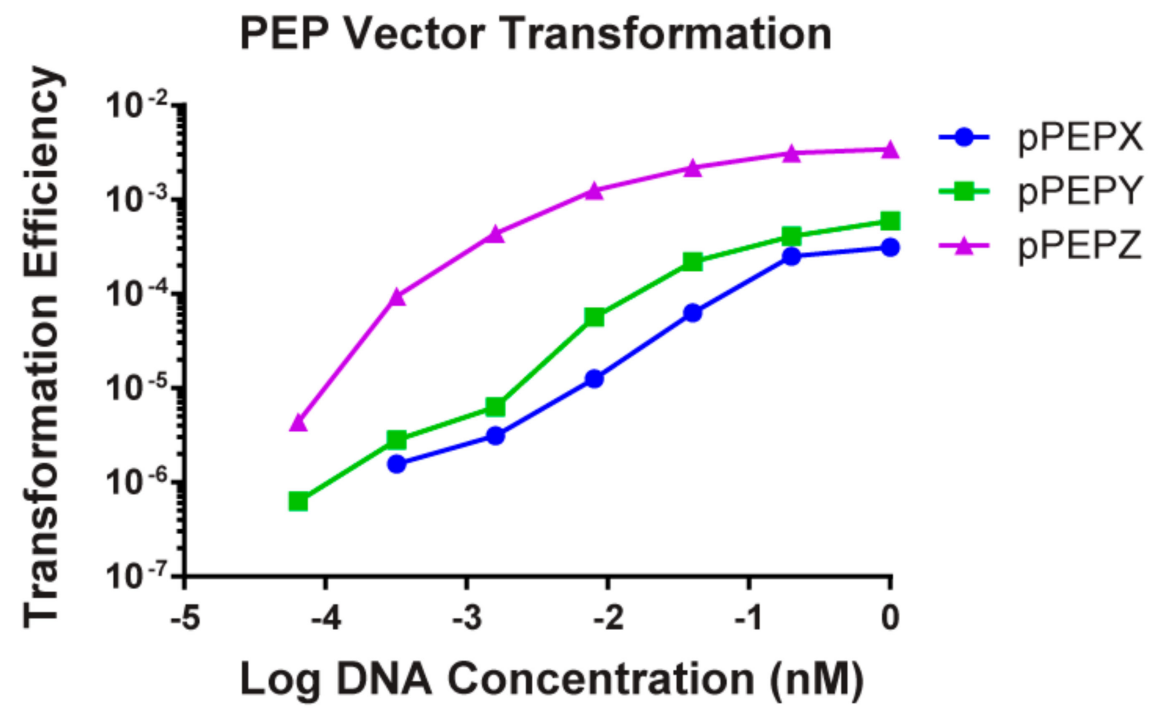

Figure 3. Transformation efficiencies of the various PEP vectors. The three PEP vectors, pPEPX, pPEPY, and pPEPZ were tested for transformation into the S. pneumoniae strain D39V. All vectors were transformable into D39V with a dose dependent response based on the amount of DNA added up to a saturation point. At all concentrations of DNA, pPEPZ had the highest transformation efficiency with pPEPX and pPEPY displaying similar rates of transformation and homologous recombination.

3.3. Simultaneous Integration and Expression of Three New Fluorescent Proteins for S. pneumoniae from the pPEPX, $p$ PEPY and $p$ PEPZ Loci

To demonstrate the feasibility to clone and construct a strain that expresses heterologous proteins from all three integration platforms simultaneously, we utilized several recent spectrally distinct fluorescent proteins not yet widely used in the pneumococcal research field, namely mTurquoise2 [22], mNeonGreen [23], and mScarlet-I [24]. To make sure these proteins do not demonstrate any spectral overlap under our experimental conditions, we fused them to genes encoding proteins with a distinct cellular localization. We cloned the genes encoding SPV_1159-mScarlet-I in pPEPX, ComEA-mTurquoise2 in pPEPY and HlpA-mNeonGreen in pPEPZ and then transformed them into strain D39V in serial (see materials and methods. Note that we directly transformed the ligation mixtures to S. pneumoniae without an intermediate E. coli clone [30].

Expression of all fusion proteins in constructed strain VL2706 was induced by addition of $1 \mathrm{mM}$ IPTG for $1 \mathrm{~h}$ before visualization by fluorescence microscopy. We selected ComEA as this is a protein involved in DNA uptake and was previously shown to demonstrate a septal localization [31]. HlpA (HU) was chosen as it binds to the nucleoid and is a good marker for the chromosome [32,33]. SPV_1159 was selected as it is a predicted small membrane protein with two transmembrane domains with both the $\mathrm{N}$ - and C-terminus inside the cytoplasm and was previously shown to demonstrate homogenous membrane localization in our laboratory (Gallay and Veening, unpublished work). As shown in Figure 4, cellular morphology of this triple labelled strain is indistinguishable from wild type and we obtained clear, distinguishable fluorescent signals for all three fusion proteins upon IPTG induction. In addition, all fusions demonstrated their predicted localization. In addition, growth curves of parent strain D39V and VL2706 were indistinguishable after $12 \mathrm{~h}$ of growth indicating that integration and the expression of genes from the integration sites did not affect growth (Supplementary Figure S2). This demonstrates that stable integration and simultaneous expression of heterologous proteins from pPEPX, pPEPY, and pPEPZ is possible, and provides a new set of fluorescent proteins for use in S. pneumoniae. 

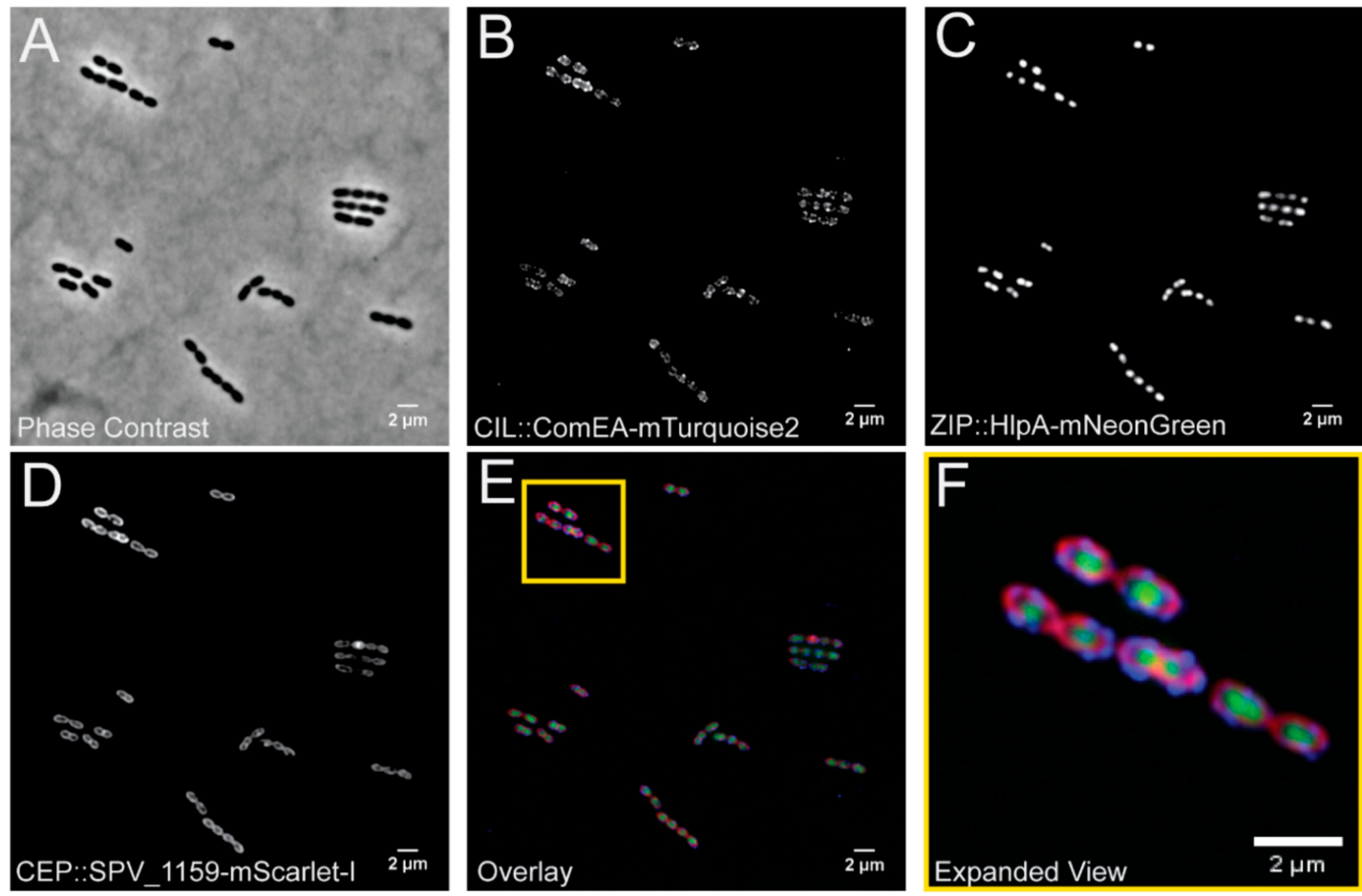

Figure 4. Visualization of a pneumococcal strain VL2706 expressing three different fluorescent fusions simultaneously from the three different integration loci. From the CEP locus (PEPX): ComEA-mTurquoise2; from the CIL locus (PEPY): SPV_1159-mScarlet-I; and from the ZIP locus (PEPZ): HlpA-mNeonGreen. Integration of the three constructed vectors produced distinct localizations based on the proteins used for the fusion. (A) A phase contrast image of VL2706. (B) The ComEA-mTurquoise2 fusion produced strong foci around midcell as well as at the poles of the cell as previously shown [31], (C) HlpA fusions produce distinct green signal that localizes at the chromosome [32,33], and (D) SPV_1159 fusions produce distinct membrane associated signals in the bacterial cells. (E) An overlay of the three simultaneously expressed fusion proteins and a yellow outline of (F) containing an expanded view of the image.

\subsection{Broad Pneumococcal Strain Range for $p P E P Y$ and $p P E P Z$}

All vectors were designed and based on the genome and transcription data of S. pneumoniae strain D39V $[13,14,34]$. To determine if the constructed vectors can be used in multiple strains of S. pneumoniae as well as closely related species, whole genome sequences were queried for the integrative regions of the PEP vectors. All genomes from NCBI at contig level or above were downloaded for S. pneumoniae (2875 strains), S. pseudopneumoniae (38 strains), S. tigurinus (19 strains), S. mutans (190 strains), S. equi (259 strains), S. pyogenes (449 strains), and S. suis (1261 strains). Additional genomes for S. pneumoniae were obtained from the Maela database [35] (3085 strains) for a total of 5960 pneumococcal genomes. Only 5029 pneumococcal genomes were tested due to duplication of some sequences between the two databases (Figure 5). A positive hit for the strain was determined when both integrative regions with a homology of at least $95 \%$ were present and had at least $90 \%$ of the total integration region. The pPEPX vector passed these requirements for $253(5.03 \%)$ of S. pneumoniae strains tested. The PPEPY vector had $3237(64.37 \%)$ positive hits for S. pneumoniae. Similar results were found for the pPEPZ vector with $3229(64.21 \%)$ hits for S. pneumoniae (Figure 5). All pneumococcal genomes queried can integrate at least one of the PEP vectors available based on sequence homology. For all other streptococcal species tested there were no strains that passed our criteria, but significant homology was found in both S. pseudopneumoniae and S. tigurinus, which are more closely related to S. pneumoniae than other strains tested [36]. Interestingly, the CEP locus for pPEPX integration was the most common locus 
in other species with some positive hits also found in S. suis. Supplementary Table S2 details which strains contained positive hits for the integration regions of the PEP vectors.

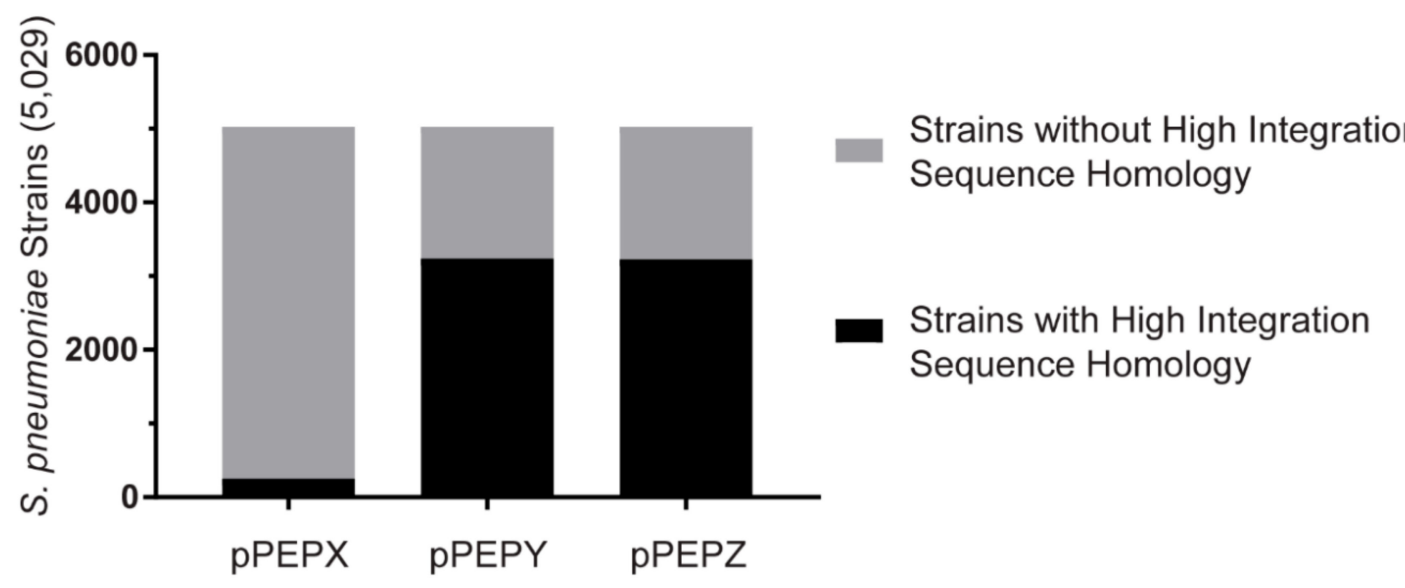

Figure 5. Frequency of $S$. pneumoniae strains with high homology to PEP vector integration regions. Local BLAST database was created containing 5029 genomes of $S$. pneumoniae. Out of these 5029 genomes, 261, 3245, and 3235 strains contained sufficient homology to the pPEPX, pPEPY, and pPEPZ vectors, respectively, to potentially for allow homologous recombination.

Testing for transformation efficiency in numerous strains and species with multiple vectors is tedious and time consuming and testing a small portion of collected strains will not be as beneficial. Although competence for transformation is highly conserved in S. pneumoniae and most strains can be transformed in the laboratory, significant differences in transformation efficiencies of different strains are observed [37]. Through using sequencing data, it is possible to test thousands of strains for the presence of the integrative regions of the constructed plasmids. While this is not conclusive evidence for the ability of the PEP vectors to be transformable into queried strains, it is indicative that they are transformable. Through using stringent cut off conditions of at least $95 \%$ homology to the integration regions and the queried strains having $90 \%$ or more of the homology region, the chances of successful genomic integration are high. Supplementary Table S3 list all pneumococcal strains that passed our criteria and indicates possible transformability with the PEP vectors.

\subsection{Efficient Cloning in Multidrug Resistant Clinical Isolates Using PEP Vectors}

Antibiotic resistance is on the rise and several clinically relevant strains are now commonly found worldwide, such as many PMEN (Pneumococcal Molecular Epidemiology Network) strains [38]. To determine the feasibility of using these plasmids in multi-drug resistant clinical isolates we tested the ability of PMEN strains 1, 3, 14, 18, 28 and TIGR4 to integrate PEP plasmids. These are serotype 23F, $9 \mathrm{~V}, 19 \mathrm{~F}, 14,1$, and 4, respectively, and have different antibiotic susceptibilities. Table 2 summarizes the strains, susceptibility, and transformability based on sequence and the experiment. All strains passed our transformability requirements based on genome sequence for at least one PEP vector. Supplementary Figures S3-S5 demonstrate the homology of the integration regions of the PEP plasmids for several clinically relevant and antibiotic resistant strains. Experimentally determined transformability with the PEP vectors verified the feasibility of using a strains' genome sequence to indicate the ability to integrate a PEP vector. Even when we did not find a high level of homology, we could still transform strains PMEN3 and PMEN14 with plasmids PPEPX and pPEPY. The exception was for the PPEPY vector, which did not yield any transformants with PMEN18 or TIGR4 despite both having high sequence homology to the integration region. 
Table 2. Transformation efficiency of PEP vectors into various pneumococcal strains. Specspectinomycin, Kan—Kanamycin, Gent-Gentamycin, Erm-Erythromycin, Tmp—Trimethoprim. *-pPEPZ plasmid chosen based on susceptibility of strain.

\begin{tabular}{ccccccc}
\hline Strain & Serotype & Susceptibility & $\begin{array}{c}\text { PEP Transformable } \\
\text { Based on Sequence }\end{array}$ & $\begin{array}{c}\text { Transformation } \\
\text { Efficiency } \\
\text { (pPEPX) }\end{array}$ & $\begin{array}{c}\text { Transformation } \\
\text { Efficiency } \\
\text { (pPEPY) }\end{array}$ & $\begin{array}{c}\text { Transformation } \\
\text { Efficiency } \\
\text { (pPEPZ }\end{array}$ \\
\hline PMEN1 & $23 \mathrm{~F}$ & Erm, Spec, Kan & pPEPY and pPEPZ & $2.56 \times 10^{-8}$ & $2.61 \times 10^{-7}$ & $8.17 \times 10^{-6}$ \\
\hline PMEN3 & $9 \mathrm{~V}$ & Erm, Spec, Kan & pPEPZ & $2.49 \times 10^{-8}$ & $3.31 \times 10^{-7}$ & $2.26 \times 10^{-8}$ \\
\hline PMEN14 & $19 \mathrm{~F}$ & Gent, Spec, Kan & pPEPZ & $8.10 \times 10^{-7}$ & $8.52 \times 10^{-7}$ & $1.43 \times 10^{-6}$ \\
\hline PMEN18 & 14 & $\begin{array}{l}\text { Gent, Spec, Kan } \\
\text { PPEPY and pPEPZ }\end{array}$ & $3.77 \times 10^{-7}$ & 0 & $2.16 \times 10^{-6}$ \\
\hline PMEN28 & 1 & $\begin{array}{c}\text { Erm, Gent, Spec, } \\
\text { Kan }\end{array}$ & N/D & $8.79 \times 10^{-8}$ & $7.95 \times 10^{-8}$ & $1.03 \times 10^{-6}$ \\
\hline TIGR4 & 4 & $\begin{array}{c}\text { Erm, Gent, Spec, } \\
\text { Kan, Tmp }\end{array}$ & pPEPX and pPEPZ & $4.98 \times 10^{-6}$ & 0 & $2.42 \times 10^{-5}$ \\
\hline
\end{tabular}

\section{Discussion}

Here, we describe the creation and testing of three integration vectors for use in Streptococcus pneumoniae. These vectors can be used for a wide range of applications in the pneumococcal field; complementation of gene deletions, labeling and expression of genes of interest, and/or expressing non-native proteins. The ability to efficiently transform and express any gene of interest stably from the chromosome as a single copy from a neutral locus is an essential tool for pneumococcal research. The PEP vectors described here can potentially be used in a large portion of pneumococcal strains and be of benefit to many researchers in the pneumococcal field. All described vectors are made available through Addgene (catalog numbers pending). It is our hope that making these vectors available will facilitate the research of other groups expanding our knowledge base of S. pneumoniae.

Supplementary Materials: The following are available online at http://www.mdpi.com/2073-4425/10/5/394/s1, Figure S1: PCR verification of double crossover of PEP plasmids. Figure S2: Growth curve of parent strain D39V and VL2706, Figure S3: Comparative alignment for CEP (pPEPX) locus and its flanking regions from various S. pneumoniae strains, Figure S4: Comparative alignment for CIL (pPEPY) locus and its flanking regions from various S. pneumoniae strains, Figure S5: Comparative alignment for ZIP (pPEPZ) locus and its flanking regions from various S. pneumoniae strains, Table S1: List of oligos used for strain construction, Table S2: Table of S. pseudopneumoniae, S. tigurinus, and S. suis strains that had some homology to various PEP vectors, Table S3: List of Streptococcus pneumoniae strains tested homology to allow for chromosomal integration of the PEP vectors.

Author Contributions: Conceptualization, L.E.K., A.-S.R., and J.-W.V.; methodology, L.E.K., A.-S.R., and J.-W.V.; validation, L.E.K., A.-S.R., and J.K.; formal analysis, L.E.K. and J.K.; investigation, L.E.K., A.-S.R., and J.K.; resources, J.-W.V.; writing-original draft preparation, L.E.K., A.-S.R., and J.K.; writing-review and editing, L.E.K., A.-S.R., J.K., and J.-W.V.; visualization, L.E.K., A.-S.R., and J.K.; supervision, J.-W.V.; funding acquisition, L.E.K. and J.-W.V.

Funding: Work in the Veening lab is supported by Defense Advanced Research Projects Agency (DARPA) cooperative agreement (W911NF-17-2-0132), by the Swiss National Science Foundation (SNSF) (project grant 31003A_172861), a JPIAMR grant (40AR40_185533) from SNSF and ERC consolidator grant 771534-PneumoCaTChER.

Conflicts of Interest: The authors declare no conflict of interest.

\section{References}

1. Bryce, J.; Boschi-Pinto, C.; Shibuya, K.; Black, R.E. WHO estimates of the causes of death in children. Lancet 2005, 365, 1147-1152. [CrossRef]

2. Khalil, A.S.; Collins, J.J. Synthetic biology: Applications come of age. Nat. Rev. Genet. 2010, 11, 367-379. [CrossRef] [PubMed]

3. Troeger, C.; Blacker, B.; Khalil, I.A.; Rao, P.C.; Cao, J.; Zimsen, S.R.M.; Albertson, S.B.; Deshpande, A.; Farag, T.; Abebe, Z.; et al. Estimates of the global, regional, and national morbidity, mortality, and aetiologies of lower respiratory infections in 195 countries, 1990-2016: A systematic analysis for the Global Burden of Disease Study 2016. Lancet Infect. Dis. 2018, 18, 1191-1210. [CrossRef] 
4. Robertson, G.T.; Ng, W.-L.; Foley, J.; Gilmour, R.; Winkler, M.E. Global transcriptional analysis of clpP mutations of type 2 Streptococcus pneumoniae and their effects on physiology and virulence. J. Bacteriol. 2002, 184, 3508-3520. [PubMed]

5. Halfmann, A.; Hakenbeck, R.; Brückner, R. A new integrative reporter plasmid for Streptococcus pneumoniae. FEMS Microbiol. Lett. 2007, 268, 217-224.

6. Eberhardt, A.; Wu, L.J.; Errington, J.; Vollmer, W.; Veening, J.-W. Cellular localization of choline-utilization proteins in Streptococcus pneumoniae using novel fluorescent reporter systems. Mol. Microbiol. 2009, 74, 395-408. [CrossRef] [PubMed]

7. Overkamp, W.; Beilharz, K.; Detert Oude Weme, R.; Solopova, A.; Karsens, H.; Kovács, Á.T.; Kok, J.; Kuipers, O.P.; Veening, J.-W. Benchmarking various green fluorescent protein variants in bacillus subtilis, Streptococcus pneumoniae, and Lactococcus lactis for live cell imaging. Appl. Environ. Microbiol. 2013, 79, 6481-6490. [CrossRef] [PubMed]

8. Wong, A.; Grau, M.A.; Singh, A.K.; Woodiga, S.A.; King, S.J. The role of neuraminidase-producing bacteria in exposing cryptic carbohydrate receptors for Streptococcus gordonii adherence. Infect. Immun. 2018, 86, e00068-18. [CrossRef]

9. Dalia, A.B.; Standish, A.J.; Weiser, J.N. Three surface exoglycosidases from Streptococcus pneumoniae, NanA, BgaA, and $\mathrm{StrH}$, promote resistance to opsonophagocytic killing by human neutrophils. Infect. Immun. 2010, 78, 2108-2116. [CrossRef]

10. King, S.J.; Hippe, K.R.; Weiser, J.N. Deglycosylation of human glycoconjugates by the sequential activities of exoglycosidases expressed by Streptococcus pneumoniae. Mol. Microbiol. 2006, 59, 961-974. [CrossRef]

11. Guiral, S.; Henard, V.; Laaberki, M.-H.; Granadel, C.; Prudhomme, M.; Martin, B.; Claverys, J.-P. Construction and evaluation of a chromosomal expression platform (CEP) for ectopic, maltose-driven gene expression in Streptococcus pneumoniae. Microbiology 2006, 152, 343-349. [CrossRef] [PubMed]

12. Sorg, R.A.; Kuipers, O.P.; Veening, J.-W. Gene expression platform for synthetic biology in the human pathogen Streptococcus pneumoniae. ACS Synth. Biol. 2014, 4, 228-239. [CrossRef]

13. Aprianto, R.; Slager, J.; Holsappel, S.; Veening, J.-W. High-resolution analysis of the pneumococcal transcriptome under a wide range of infection-relevant conditions. Nucleic Acids Res. 2018, 46, 9990-10006. [CrossRef]

14. Slager, J.; Aprianto, R.; Veening, J.-W. Deep genome annotation of the opportunistic human pathogen Streptococcus pneumoniae D39. Nucleic Acids Res. 2018, 46, 9971-9989. [CrossRef] [PubMed]

15. Domenech, A.; Slager, J.; Veening, J.-W. Antibiotic-induced cell chaining triggers pneumococcal competence by reshaping quorum sensing to autocrine-like signaling. Cell Rep. 2018, 25, 2390-2400. [CrossRef]

16. Martin, B.; Garcia, P.; Castanié, M.; Claverys, J. The recA gene of Streptococcus pneumoniae is part of a competence-induced operon and controls lysogenic induction. Mol. Microbiol. 1995, 15, 367-379. [CrossRef]

17. Slager, J.; Kjos, M.; Attaiech, L.; Veening, J.-W. Antibiotic-induced replication stress triggers bacterial competence by increasing gene dosage near the origin. Cell 2014, 157, 395-406. [CrossRef]

18. Sorg, R.A. Engineering Approaches to Investigate Pneumococcal Gene Expression Regulation and Antibiotic Resistance Development; Rijksuniversiteit: Groningen, The Netherlands, 2016; ISBN 9036792584.

19. Fischer, K.E.; Bremer, E. Activity of the osmotically regulated yqiHIK promoter from Bacillus subtilis is controlled at a distance. J. Bacteriol. 2012, 194, 5197-5208. [CrossRef]

20. Liu, X.; Gallay, C.; Kjos, M.; Domenech, A.; Slager, J.; van Kessel, S.P.; Knoops, K.; Sorg, R.A.; Zhang, J.; Veening, J. High-throughput CRISPRi phenotyping identifies new essential genes in Streptococcus pneumoniae. Mol. Syst. Biol. 2017, 13, 931. [CrossRef] [PubMed]

21. Minnen, A.; Attaiech, L.; Thon, M.; Gruber, S.; Veening, J. SMC is recruited to oriC by ParB and promotes chromosome segregation in Streptococcus pneumoniae. Mol. Microbiol. 2011, 81, 676-688. [CrossRef]

22. Goedhart, J.; von Stetten, D.; Noirclerc-Savoye, M.; Lelimousin, M.; Joosen, L.; Hink, M.A.; van Weeren, L.; Gadella, T.W.J. Jr.; Royant, A. Structure-guided evolution of cyan fluorescent proteins towards a quantum yield of 93\%. Nat. Commun. 2012, 3, 751. [CrossRef]

23. Shaner, N.C.; Lambert, G.G.; Chammas, A.; Ni, Y.; Cranfill, P.J.; Baird, M.A.; Sell, B.R.; Allen, J.R.; Day, R.N.; Israelsson, M. A bright monomeric green fluorescent protein derived from Branchiostoma lanceolatum. Nat. Methods 2013, 10, 407-409. [CrossRef] 
24. Bindels, D.S.; Haarbosch, L.; van Weeren, L.; Postma, M.; Wiese, K.E.; Mastop, M.; Aumonier, S.; Gotthard, G.; Royant, A.; Hink, M.A.; et al. mScarlet: A bright monomeric red fluorescent protein for cellular imaging. Nat. Methods 2016, 14, 53-56. [CrossRef] [PubMed]

25. Ohtsubo, Y.; Ikeda-Ohtsubo, W.; Nagata, Y.; Tsuda, M. GenomeMatcher: A graphical user interface for DNA sequence comparison. BMC Bioinform. 2008, 9, 376. [CrossRef]

26. Rasband, W.S. ImageJ. US National Institutes of Health; Bethesda: Rockville, MD, USA, 1997.

27. Anderson, J.; Dueber, J.E.; Leguia, M.; Wu, G.C.; Goler, J.A.; Arkin, A.P.; Keasling, J.D. BglBricks: A flexible standard for biological part assembly. J. Biol. Eng. 2010, 4, 1. [CrossRef]

28. Lau, P.C.Y.; Sung, C.K.; Lee, J.H.; Morrison, D.A.; Cvitkovitch, D.G. PCR ligation mutagenesis in transformable streptococci: Application and efficiency. J. Microbiol. Methods 2002, 49, 193-205. [CrossRef]

29. Lee, M.S.; Seok, C.; Morrison, D.A. Insertion-duplication mutagenesis in Streptococcus pneumoniae: Targeting fragment length is a critical parameter in use as a random insertion tool. Appl. Environ. Microbiol. 1998, 64, 4796-4802. [PubMed]

30. Beilharz, K.; van Raaphorst, R.; Kjos, M.; Veening, J.-W. Red fluorescent proteins for gene expression and protein localization studies in Streptococcus pneumoniae and efficient transformation with Gibson assembled DNA. Appl. Environ. Microbiol. 2015, 81, 7244-7252. [CrossRef] [PubMed]

31. Bergé, M.J.; Kamgoué, A.; Martin, B.; Polard, P.; Campo, N.; Claverys, J.-P. Midcell recruitment of the DNA uptake and virulence nuclease, EndA, for pneumococcal transformation. PLoS Pathog. 2013, 9, e1003596. [CrossRef]

32. Kjos, M.; Aprianto, R.; Fernandes, V.E.; Andrew, P.W.; van Strijp, J.A.G.; Nijland, R.; Veening, J.-W. Bright fluorescent Streptococcus pneumoniae for live-cell imaging of host-pathogen interactions. J. Bacteriol. 2015, 197, 807-818. [CrossRef]

33. Kjos, M.; Veening, J. Tracking of chromosome dynamics in live Streptococcus pneumoniae reveals that transcription promotes chromosome segregation. Mol. Microbiol. 2014, 91, 1088-1105. [CrossRef] [PubMed]

34. Aprianto, R.; Slager, J.; Holsappel, S.; Veening, J.-W. Time-resolved dual RNA-seq reveals extensive rewiring of lung epithelial and pneumococcal transcriptomes during early infection. Genome Biol. 2016, 17, 198. [CrossRef] [PubMed]

35. Chewapreecha, C.; Harris, S.R.; Croucher, N.J.; Turner, C.; Marttinen, P.; Cheng, L.; Pessia, A.; Aanensen, D.M.; Mather, A.E.; Page, A.J. Dense genomic sampling identifies highways of pneumococcal recombination. Nat. Genet. 2014, 46, 305-309. [CrossRef] [PubMed]

36. Gao, X.-Y.; Zhi, X.-Y.; Li, H.-W.; Klenk, H.-P.; Li, W.-J. Comparative genomics of the bacterial genus Streptococcus illuminates evolutionary implications of species groups. PLoS ONE 2014, 9, e101229. [CrossRef] [PubMed]

37. Evans, B.A.; Rozen, D.E. Significant variation in transformation frequency in Streptococcus pneumoniae. ISME J. 2013, 7, 791-799. [CrossRef] [PubMed]

38. McGee, L.; McDougal, L.; Zhou, J.; Spratt, B.G.; Tenover, F.C.; George, R.; Hakenbeck, R.; Hryniewicz, W.; Lefevre, J.C.; Tomasz, A. Nomenclature of major antimicrobial-resistant clones of Streptococcus pneumoniae defined by the pneumococcal molecular epidemiology network. J. Clin. Microbiol. 2001, 39, 2565-2571. [CrossRef] [PubMed]

(C) 2019 by the authors. Licensee MDPI, Basel, Switzerland. This article is an open access article distributed under the terms and conditions of the Creative Commons Attribution (CC BY) license (http://creativecommons.org/licenses/by/4.0/). 